\title{
Quantitative assessment of myocardial mechanics in patients with cardiac amyloid using cardiovascular magnetic resonance myocardial feature tracking
}

Shazia T Hussain ${ }^{2 *}$, Sebastian Buss ${ }^{3}$, Shelby Kutty ${ }^{4}$, Henning Steen ${ }^{3}$, Dirk Lossnitzer ${ }^{3}$, Philipp B Beerbaum ${ }^{5}$, Pablo Lamata ${ }^{1}$, Andreas Schuster ${ }^{6}$

From 18th Annual SCMR Scientific Sessions

Nice, France. 4-7 February 2015

\section{Background}

Cardiovascular magnetic resonance (CMR) feature-tracking (FT) allows the quantitative assessment of complex ventricular mechanics such as strain, twist and untwist. LV twist results from the dynamic interplay between systolic clockwise rotation of the base and a counterclockwise rotation of the apex followed by untwisting during diastole.

We sought to determine whether strain, myocardial twist and untwist rates could be measured by CMR-FT and hypothesized that twist and untwist rates would be reduced in patients with amyloid disease as a consequence of systolic and diastolic dysfunction.

\section{Methods}

The CMR images of 62 patients with biopsy-proven amyloid, and 10 healthy volunteers were assessed with CMR-FT post-processing software (TomTec, Germany). All subjects had routine steady state free precession (SSFP) cine imaging in the short axis and 4-chamber orientations at 1.5 Tesla. Peak longitudinal (Ell), radial

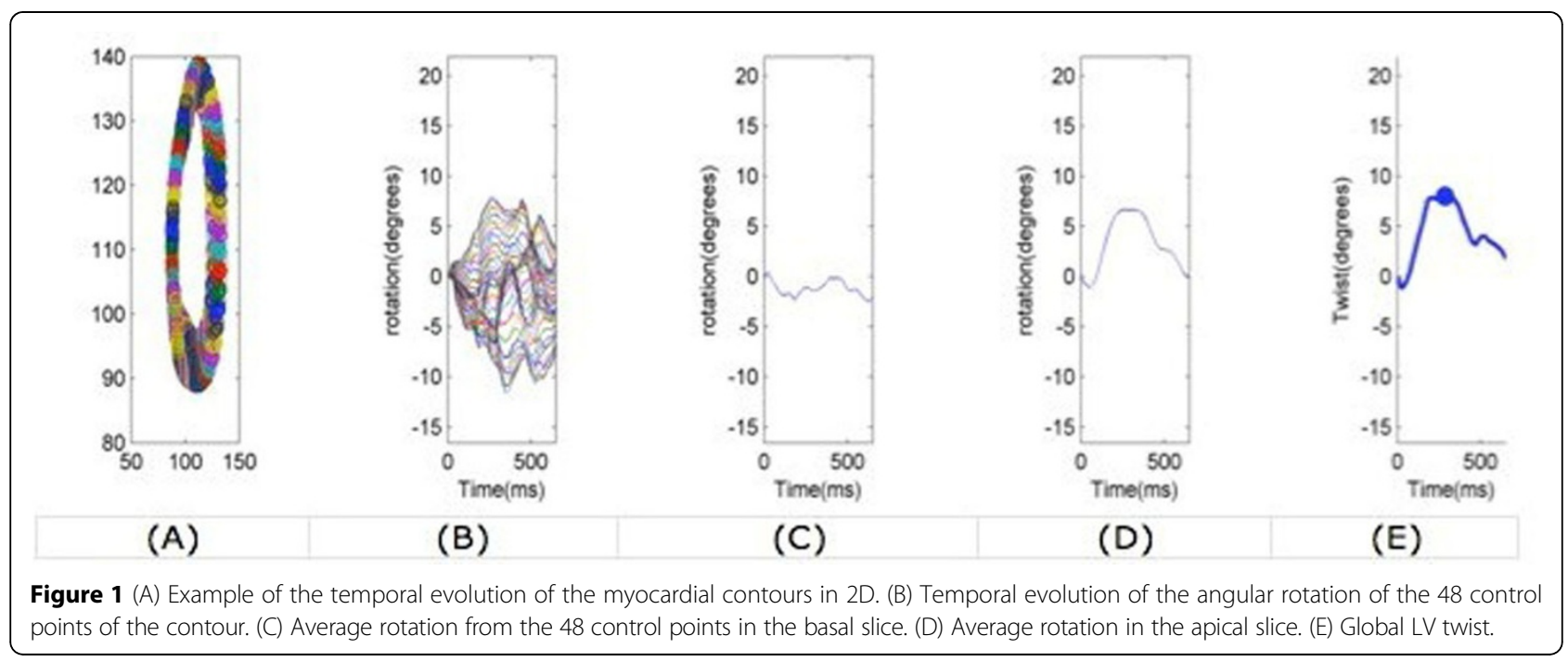

${ }^{2}$ Cardiology, Papworth Hospital, Cambridgeshire, UK

Full list of author information is available at the end of the article

(c) 2015 Hussain et al; licensee BioMed Central Ltd. This is an Open Access article distributed under the terms of the Creative Commons 
Table 1 Mean values of peak strain, peak twist and untwist rates in the amyloid cohort and in volunteers

\begin{tabular}{|c|c|c|c|}
\hline Strain/Twist & Group & Mean value - peak \% (+/-SD) & Sig (2 tailed)* \\
\hline \multirow[t]{2}{*}{$\overline{E l l}$} & Volunteer & $-18.78(+/-3.7)$ & $p<0.005$ \\
\hline & Amyloid & $-6.73(+/-11)$ & \\
\hline \multirow[t]{2}{*}{ Err } & Volunteer & $36.97(+/-26.5)$ & $p=0.510$ \\
\hline & Amyloid & $35.00(+/-35)$ & \\
\hline \multirow[t]{2}{*}{ Ecc endo } & Volunteer & $-25.96(+/-11.6)$ & $p<0.005$ \\
\hline & Amyloid & $-17.81(+/-11.4)$ & \\
\hline \multirow[t]{2}{*}{ Ecc epi } & Volunteer & $-17.26(+/-9.6)$ & $p<0.005$ \\
\hline & Amyloid & $-10.34(+/-9.3)$ & \\
\hline \multirow[t]{2}{*}{ Twist(degrees) } & Volunteer & $8.05(+/-6.1)$ & $p=0.025$ \\
\hline & Amyloid & $3.05(+/-6.42)$ & \\
\hline \multirow[t]{2}{*}{ Untwist-rate (degrees/s) } & Volunteer & $-65.5(+/-46.42)$ & $P=0.04$ \\
\hline & Amyloid & $-19.2(+/-45.6)$ & \\
\hline
\end{tabular}

* Significance tested with an unpaired t-test

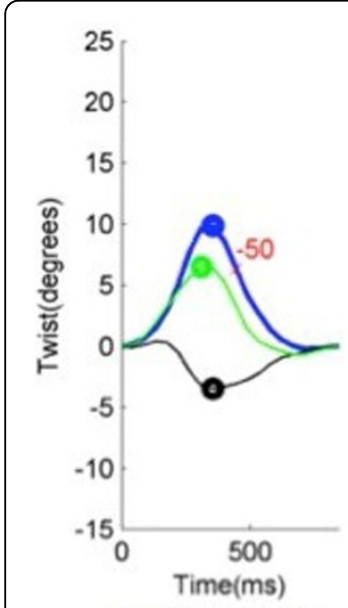

(A) Volunteer

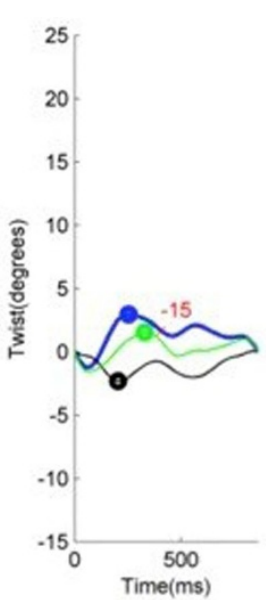

(B) Amyloid patient
Figure 2 Overall twist. Overall twist (purple line) is calculated from the difference between counterclockwise apical rotation (green line) and the clockwise basal rotation (black line). The untwist rate is marked in red. The results in the amyloid pt (B) are reduced compared to those of the volunteer (A)

(Err) and circumferential endocardial $\left(\mathrm{Ecc}_{\text {endo }}\right)$ and circumferential epicardial $\left(\mathrm{Ecc}_{\mathrm{epi}}\right)$ strain was measured. Additionally, the rotation of the basal and apical slices was measured and global LV twist $\theta$ was calculated as the difference between the overall counterclockwise (positive) rotation at the apex $\left(\phi_{\text {apex }}\right)$ and the overall clockwise rotation at the base (viewed from apex), $\theta=$ $\phi_{\text {apex }}-\phi_{\text {base }}$.

Peak twist and untwist rates were calculated using MATLAB software (figure 1).

\section{Results}

We observed a significant difference in myocardial twist and the untwist rate between the patients and the volunteers $(\mathrm{p}<0.05$, table 1 and figure 2$)$. Whilst there was a significant reduction in longitudinal and circumferential strain in the amyloid cohort compared to the volunteers $(\mathrm{p}<0.05$, table 1$)$ the radial strain remained unchanged $(\mathrm{p}>0.05$, table 1$)$.

\section{Conclusions}

This study demonstrates the feasibility of measuring complex mechanics from routine cine images in patients with amyloid using CMR-FT.

The reduction in strain and twist in the amyloid patients is likely to reflect systolic dysfunction. In addition reduced untwist rates may be a measure of diastolic dysfunction.

Larger scale studies are required to validate this further, however, we have demonstrated that CMR-FT has the potential to define diastolic dysfunction from routinely acquired CMR cine images.

\section{Funding}

N/A.

\section{Authors' details}

${ }^{1}$ Imaging Sciences, Kings College Hospital, London, UK. ${ }^{2}$ Cardiology, Papworth Hospital, Cambridgeshire, UK. ${ }^{3}$ Cardiology, University of Heidelberg, Heidelberg, Germany. ${ }^{4}$ University of Nebraska, Omaha, NE. ${ }^{5}$ Department of paediatric cardiology, Hannover Medical School, Hannover, Germany. ${ }^{6}$ Cardiology and Pneumology, Georg-August-University, Gottingen, Germany.

Published: 3 February 2015

doi:10.1186/1532-429X-17-S1-Q28

Cite this article as: Hussain et al:: Quantitative assessment of myocardial mechanics in patients with cardiac amyloid using cardiovascular magnetic resonance myocardial feature tracking. Journal of Cardiovascular Magnetic Resonance 2015 17(Suppl 1):Q28. 